\title{
Mental Wellbeing Monitoring in a Sample of Emergency Medical Service Personnel
}

\author{
Charles Van Wijk¹, Frans Cronje², Jack Meintjes,3 \\ ${ }^{1}$ Private, Simon's Town, South Africa \\ ${ }^{2}$ Hyperbaric Medicine Unit, Emergency Medical Services, Cape Town, South Africa \\ ${ }^{3}$ Division of Health Systems and Public Health, Department of Global Health, Stellenbosch University, Cape Town, South Africa \\ ${ }^{4}$ Tygerberg Academic Hospital, Occupational Health Department, Cape Town, South Africa \\ Email:chvanwijk@gmail.com,fjcgeneral@gmail.com,wasjm@sun.ac.za
}

How to cite this paper: Van Wijk, C., Cronje, F. and Meintjes, J. (2020) Mental Wellbeing Monitoring in a Sample of Emergency Medical Service Personnel. Occupational Diseases and Environmental Medicine, 8, 26-33. https://doi.org/10.4236/odem.2020.81002

Received: December 29, 2019

Accepted: February 18, 2020

Published: February 21, 2020

Copyright $\odot 2020$ by author(s) and Scientific Research Publishing Inc. This work is licensed under the Creative Commons Attribution International License (CC BY 4.0).

http://creativecommons.org/licenses/by/4.0/ (c) (i) Open Access

\begin{abstract}
Background: There have been concerns regarding the mental wellbeing of emergency medical service staff, particularly when faced with multiple trauma exposures. This study aimed to describe a cross-sectional view of the mental wellbeing status of emergency medical service workers, as well as to comment on the usefulness of an electronic survey tool to reach staff that is distributed across large geographical areas. Methods: The data presented here are drawn from an anonymous, voluntary, electronic survey, made available via the internet, to emergency medical service workers in the Western Cape province of South Africa. This paper reports on four domain expressions of mental wellbeing, namely disordered mood, problematic substance use, post-traumatic stress disorder, and domestic discord. Results: Elevated rates were reported by this sample, across all four distress domains, i.e. mood disorder $=12.3 \%$; problematic substance use $=14.9 \%$; post-traumatic stress disorder $=11.9 \%$; and domestic discord $=11.9 \%$. These rates were generally similar to data from comparable emergency worker groups, and much higher than local population estimates. Conclusions: The data support earlier findings that emergency medical work is associated with increased risk for symptoms of psychological distress, which present a strong case for regular organizational monitoring of employees' mental wellbeing. In this regard, a brief, internet delivered, survey-type tool showed promise for screening employees, in order to guide further streaming of distressed individuals towards appropriate support services. Further work is required to develop clear mechanisms for referral and intervention in order to optimize a confidential and supportive monitoring program.
\end{abstract}

\section{Keywords}

Emergency Medical Service Employees, Internet Screening, Mental 
Wellbeing, Psychological Distress

\section{Introduction}

Emergency medical services (EMS) work can have significant emotional costs, with reports indicating elevated psychiatric symptoms and psychological distress as a result of work exposure [1], with repeated exposure to suffering and trauma among the factors contributing to this elevated risk [2]. Post-traumatic stress disorder (PTSD) has been a main focus of research on the mental health of this population, with prevalence rates around $11 \%$ reported for emergency service workers [1] [3]. Ambulance personnel are at particularly high risk, reporting significantly higher rates of PTSD compared to other emergency services such as police and firefighters [3]. Other possible mental health consequences of regular trauma and stress in emergency medical work are less well studied. Reported rates of depression (15\%), anxiety (15\%), and general psychological distress (27\%) in ambulance personnel are higher than population estimates [1], with increasing rates of depression and anxiety observed as the burden of cumulative trauma increases [4].

Reports from South African (SA) EMS indicate greater exposure to critical incidents by staff than comparable groups in the developed world [5], associated with a range of adverse outcomes, with staff reporting problematic alcohol use (22.5\%), PTSD (2.4\%), psychological aggression (4.5\%), and physical assault (1.9\%) [5]. Further, South Africans are exposed to widespread community trauma [6], and EMS staff also face additional, potentially traumatic work-related stressors that include direct criminal attacks on staff members while responding to medical emergencies.

Concerns about the risk to SA EMS staff of developing a range of post-traumatic stress responses after exposure to suffering and trauma, especially in the local context of multiple exposures, emphasized the need for the monitoring of mental wellbeing of employees. Against this background, a project was initiated by the Western Cape (WC) EMS, with the aim of exploring mechanisms around mental wellbeing surveillance in support of employees.

This report aims to describe three aspects of the project, namely:

1) To provide a descriptive, cross-sectional view of the current mental wellbeing status of WC EMS staff during October 2018;

2) To comment on an electronic survey-type tool to provide access to all staff members, who are distributed across a large geographical province; and

3) To consider the screen-to-stream concept to support employee wellbeing, i.e. the use of survey type screening to inform streaming of distressed individuals towards appropriate support services.

The report will present screening data across four domains of potential psychological distress after traumatic exposure, namely mood disorders, proble- 
matic substance use, PTSD, and domestic discord.

\section{Materials and Methods}

\subsection{Sample}

The data presented here were drawn from an anonymous, voluntary, electronic survey, made available to all WC EMS personnel during October 2018 (designed to co-inside with mental health month), and who were invited to complete it at their own initiative within the month-long time-period.

\subsection{Measuring Tool}

Responses were collected using an internet-based survey-type tool, which included items that tapped into symptoms from a range of syndromes associated with post-trauma stress reactions. The full length survey comprised items from DSM-5 syndromes [7], including mood and post-traumatic stress disorders, as well as other short validated scales. The value of short screening scales in low and middle income countries have convincingly been demonstrated [8], and persuasive validation of DSM-5 self-rated screeners specifically have also been reported [9] [10]. Only results from the four domains of potential psychological distress mentioned above are reported here.

The mood domain comprised items for the DSM-5 Major Depressive Disorder criteria [7], which cover the two core depressive symptoms (depressed mood and anhedonia), as well as somatic symptoms and negative cognitions. A score of 5 was used to determine a limited DSM- 5 criteria threshold to indicate a conservative estimation of cases of concern. A score of 4 suggested a need for further assessment.

Problematic alcohol use was determined using the validated 4-item CAGE [11] [12], with a score $\geq 2$ indicative of concern. A score of 1 suggested a need for further assessment. Problematic drug use was determined by a positive response to the validated Single-Question Screening Test for Drug Use in Primary Care scale [13].

The PTSD domain comprised items for the DSM-5 Post-traumatic Stress Disorder criteria [7], which cover intrusive symptoms, avoidance, negative alterations in cognition and mood, and alterations in arousal. Diagnostic likelihood was determined using two methods. Firstly, a score $\geq 3$ on the validated PC-PTSD-5 [14] were considered indicative of concern. Secondly, an expanded formula based on DSM-5 criteria [7] was used to indicate a more conservative estimate (i.e. score $\geq 6$ ). Participants answered questions specifically on work-related PTSD, and further indicated exposure to non-work-related trauma. If they answered yes to the latter, they were directed to answer similar PTSD questions within the non-work-related context.

Possible work-home spill-over were determined by three items reflecting domestic discord, where a score of 3 was considered a conservative indicator of work exposure influencing relations at home. A score of 2 suggested need for 
further assessment. This scale was developed specifically for the study.

The survey was introduced to the participants under the title of Mental Wellness. The survey introduction made the explicit link to "mental health in emergency medical services", and how work-related experiences of staff may impact on their mental wellbeing. The survey items were worded to imply both a change from previous functioning, and the unpleasant nature of the symptoms.

The survey was further designed to provide immediate feedback to participants. Summed scores in each domain triggered feedback options of either reassurance, or encouragement to make contact with their general practitioners or work supervisors, whichever was the most appropriate to the context. Feedback occurred at the end of each domain's items.

\subsection{Interpretation of Responses}

Although the link to work exposure was implied, direct causality of symptoms to work exposure could not be assumed, except in the case of work-related PTSD, which made specific reference to work events.

Symptoms were calculated and compared to guidelines (e.g. minimum requirements for diagnostic likelihood), to indicate cases of concern. Although different interpretative frameworks were available, only two sets of findings are reported here. The first used the most conservative estimates, indicating the highest likelihood of meeting diagnostic thresholds, while the second used the criteria for referral for further assessment. This is presented in Table 1.

\section{Results}

Through the month-long survey, 268 usable responses were obtained ( $\pm 31 \%$ response rate). This met minimum sample size requirements, which allowed further analyses. Of the participants, 3.6\% were registered with the Medical \& Dental Board (of the HPCSA), 63.5\% with the Emergency Care Board (of the HPCSA), 3.1\% were registered with other HPCSA Boards, and $29.8 \%$ were not registered with the HPCSA (e.g. Call Centre staff). Responses were received from

Table 1. Outcomes of survey responses across mental wellbeing domains.

\begin{tabular}{cccc}
\hline Diagnostic category & $\begin{array}{c}\text { Number of } \\
\text { completed } \\
\text { responses }\end{array}$ & $\begin{array}{c}\text { Met criteria } \\
\text { suggesting current } \\
\text { diagnosis }\end{array}$ & $\begin{array}{c}\text { Met criteria for } \\
\text { referral for further } \\
\text { assessment }\end{array}$ \\
\cline { 2 - 4 } & $\mathrm{N}$ & $(\mathrm{n}) / \%$ & (n) $/ \%$ \\
\hline PTSD - work related & 243 & $(29) 11.9 \%$ & (50) $20.6 \%$ \\
PTSD - not work related & 75 & (11) $4.5 \%$ & (24) $9.9 \%$ \\
Mood (depression) & 243 & (30) $12.3 \%$ & (51) $19.0 \%$ \\
Problematic alcohol use & 249 & (37) $14.9 \%$ & (58) $23.3 \%$ \\
Drug misuse & 249 & (34) $13.7 \%$ & \\
Domestic discord & 236 & $(28) 11.9 \%$ & (54) $22.9 \%$ \\
\hline
\end{tabular}


nine different geographical locations.

Conservatively, when using DSM-5 criteria, $11.9 \%$ of the sample reported symptoms suggesting current PTSD associated with work-related traumatic exposure. Further, $4.5 \%$ of the sample reported symptoms suggesting current PTSD related to traumatic exposure outside of work, and which constituted a $63.6 \%$ overlap with work-related PTSD. In total, $32 \%$ of the sample reported exposure to both work- and non-work-related trauma.

Additionally, when applying both DSM-5 symptom and time criteria, 12.3\% of the sample reported symptoms suggesting current major depressive disorder. Further, $14.9 \%$ of the sample indicated possible problematic alcohol use (based on CAGE responses), while $13.7 \%$ indicated problematic drug use, with an overlap of $29.4 \%$ between alcohol and drug (mis) use. Domestic discord, as indicator of work-stress spilling over into home life, was reported by $11.9 \%$ of the sample.

Of the participants who met criteria for work-related PTSD, $62.1 \%$ also reported symptoms consistent with mood disorder, while $41.4 \%$ reported problematic alcohol or drug use, and $48.3 \%$ reported domestic discord. This suggests substantial co-morbidity in this sample.

\section{Discussion}

The first aim of this report was to describe a cross-sectional view of the mental wellbeing status of WC EMS staff. It is based on a relatively small sample, and some bias in who completed the survey could be assumed, in that individuals who experienced distress may have been more likely to participate. However, the outcomes reported represent extremely conservative estimates, and may reflect reasonable incidences of significant mental distress.

The $11.9 \%$ prevalence rate of PTSD in this sample were similar to international pooled EMS rates of $11 \%$ [1], and much higher than a previous report of $2.4 \%$ for WC EMS staff (which used a different measure [5]). It was also much higher than the SA 12 -month prevalence estimate of $0.6 \%$ [15]. The $12.3 \%$ prevalence rate of mood disorder in this sample were also similar to international pooled EMS rates of 15\% [1], and higher than the SA 12-month prevalence estimate of 4.9\% [15]. Reports of problematic use of alcohol/substances (namely 14.9\%/13.7\%) were generally similar to a previous report of $22.5 \%$ for WC EMS staff (which used the same measure [5]), and higher than the SA 12-month prevalence estimate of $5.8 \%$ [15]. This sample of WC EMS staff reported higher rates of PTSD, depression, and problematic substance use than other South Africans, and similar to EMS workers elsewhere.

The data also highlighted the issue of complex PTSD, in that $32 \%$ participants reported exposure to trauma both at work and outside of it. The cumulative effect of multiple trauma exposure on psychological distress in SA is well established [6], and may require careful monitoring in the EMS context to ensure adequate support is offered to high risk employees.

The data is a further reminder that post-traumatic stress can be expressed 
through various forms of compromised mental wellbeing, and is not limited to PTSD. This could be seen in the high co-morbidity of PTSD and mood (62.1\%), and PTSD and problematic substance use (41.2\%). Measures of disrupted mood, problematic substance use, and domestic discord may therefore need to be included when considering the impact of work on mental wellbeing.

The second aim was to consider an electronic survey-type tool delivered via the internet, as a mechanism to reach staff members distributed across a large geographical province. Responses from at least nine separate areas suggested wide geographical coverage of the project, and indicate promise for this type of surveillance mechanism to support employees, particularly those outside major urban centers.

The third aim was to explore possible mechanisms to respond to reported indicators of distress. Although informal reports suggest that real-time feedback was experienced positively, participants' actual responses to the feedback prompts are not known, and this limitation of the study require further research. However, the concept of a screening mechanism to enable streaming of at-risk employees towards appropriate support services remain pertinent, and could become a significant means to achieve organizational support for employees' mental wellbeing.

The study had a number of limitations: In the literature on the topic, different measures are reported across studies. Although this study used the internationally recognized DSM-5 criteria and other validated scales, the diversity of previously reported measures may limit comparison of results across various samples. The current study also did not differentiate between different professional groups within EMS, and given that their work (and thus exposure) may be different, further research is necessary to address context-specific outcomes (e.g. emergency room doctors versus ambulance personnel).

\section{Conclusions}

In conclusion, the data support earlier findings that emergency medical work is associated with elevated symptoms of psychological distress, which present a strong case for regular organizational monitoring of employees' mental wellbeing. In this regard, a brief, internet delivered, survey-type tool showed promise as a proof of concept for the screening of employees. This could in future guide further streaming of distressed individuals towards appropriate support services. The proof of concept is supported by the findings that, firstly, the internet-based survey reported outcomes similar to other studies on EMS personnel, and secondly, by the wide geographical distribution from where responses were submitted.

Further work is now required to develop clear mechanisms for referral and intervention in order to optimize a confidential and supportive monitoring program.

\section{Acknowledgements}

The authors wish to thank Dr Shaheem de Vries and Prof Lee Wallis for sup- 
porting this project.

\section{Conflicts of Interest}

The authors have no conflicts of interest to declare.

\section{References}

[1] Petrie, K., Milligan-Saville, J., Gayed, A., Deady, M., Phelps, A., Dell, L., et al. (2018) Prevalence of PTSD and Common Mental Disorders amongst Ambulance Personnel: A Systematic Review and Meta-Analysis. Social Psychiatry and Psychiatric Epidemiology, 53, 897-909. https://doi.org/10.1007/s00127-018-1539-5

[2] Mitchell, K.S., Wolf, E.J., Bovin, M.J., Lee, L.O., Green, J.D., Rosen, R.C., et al. (2017) Network Models of DSM-5 Posttraumatic Stress Disorder: Implications for ICD-11. Journal of Abnormal Psychology, 126, 355-366. https://doi.org/10.1037/abn0000252

[3] Berger, W., Coutinho, E.S.F., Figueira, I., Marques-Portella, C., Luz, M.P., Neylan, T.C., et al. (2012) Rescuers at Risk: A Systematic Review and Meta-Regression Analysis of the Worldwide Current Prevalence and Correlates of PTSD in Rescue Workers. Social Psychiatry and Psychiatric Epidemiology, 47, 1001-1011. https://doi.org/10.1007/s00127-011-0408-2

[4] Harvey, S.B., Milligan-Saville, J.S., Paterson, H.M., Harkness, E.L., Marsh, A.M., Dobson, M., et al. (2016) The Mental Health of Fire-Fighters: An Examination of the Impact of Repeated Trauma Exposure. Australian and New Zealand Journal of Psychiatry, 50, 64-58. https://doi.org/10.1177/0004867415615217

[5] Ward, C.L., Lombard, C.J. and Gwebushe, N. (2006) Critical Incident Exposure in South African Emergency Services Personnel: Prevalence and Associated Mental Health Issues. Emergency Medicine Journal, 23, 226-231. https://doi.org/10.1136/emj.2005.025908

[6] Williams, S.L., Williams, D.R., Stein, D.J., Seedat, S., Jackson, P.B. and Moomal, H. (2007) Multiple Traumatic Events and Psychological Distress: The South Africa Stress and Health Study. Journal of Traumatic Stress, 20, 845-855.

https://doi.org/10.1002/jts.20252

[7] American Psychiatric Association (2013) Diagnostic and Statistical Manual of Mental Disorders. 5th Edition, American Psychiatric Association, Arlington. https://doi.org/10.1176/appi.books.9780890425596

[8] Akena, D., Joska, J., Obuku, E.A., Amos, T., Musisi, S. and Stein, D. (2012) Comparing the Accuracy of Brief versus Long Depression Screening Instruments Which Have Been Validated in Low and Middle Income Countries: A Systematic Review. BMC Psychiatry, 12, Article No. 187. https://doi.org/10.1186/1471-244X-12-187 http://www.biomedcentral.com/1471-244X/12/187

[9] Bravo, A.J., Villarosa-Hurlocker, M.C., Pearson, M.R. and Protective Strategies Study Team (2018) College Student Mental Health: An Evaluation of the DSM-5 Self-Rated Level 1 Cross-Cutting Symptom Measure. Psychological Assessment, 30, 1382-1389. https://doi.org/10.1037/pas0000628

[10] Foa, E.B., McLean, C.P., Zang, Y., Zhong, J., Powers, M.B., Kauffman, B.Y., et al. (2016) Psychometric Properties of the Posttraumatic Diagnostic Scale for DSM-5. Psychological Assessment, 28, 1166-1171. https://doi.org/10.1037/pas0000258

[11] Ewing, J.A. (1984) Detecting Alcoholism: The CAGE Questionnaire. Journal of the American Medical Association, 252, 1905-1907. 
https://doi.org/10.1001/jama.252.14.1905

[12] Williams, N. (2014) The CAGE Questionnaire. Occupational Medicine, 64, 473-474. https://doi.org/10.1093/occmed/kqu058

[13] Smith, P.C., Schmidt, S.M., Allensworth-Davies, D. and Saitz, R.A. (2010) Single-Question Screening Test for Drug Use in Primary Care. Archives of Internal Medicine, 170, 1155-1160. https://doi.org/10.1001/archinternmed.2010.140

[14] Prins, A., Bovin, M.J., Smolenski, D.J., Mark, B.P., Kimerling, R., Jenkins-Guarnieri, M.A., et al. (2016) The Primary Care PTSD Screen for DSM-5 (PC-PTSD-5): Development and Evaluation within a Veteran Primary Care Sample. Journal of General Internal Medicine, 31, 1206-1211. https://doi.org/10.1007/s11606-016-3703-5

[15] Herman, A.A., Stein, D.J., Seedat, S., Heeringa, S.G., Mooma, H. and Williams, D.R. (2009) The South African Stress and Health (SASH) Study: 12-Month and Lifetime Prevalence of Common Mental Disorders. South African Medical Journal, 99, 339-344. https://www.ncbi.nlm.nih.gov/pmc/articles/PMC3191537 